This item was submitted to Loughborough's Research Repository by the author.

Items in Figshare are protected by copyright, with all rights reserved, unless otherwise indicated.

\title{
Charge redistribution and properties of high-temperature superconductors
}

PLEASE CITE THE PUBLISHED VERSION

PUBLISHER

(C) American Physical Society

LICENCE

CC BY-NC-ND 4.0

REPOSITORY RECORD

Khomskii, D.I., and F.V. Kusmartsev. 2019. "Charge Redistribution and Properties of High-temperature Superconductors". figshare. https://hdl.handle.net/2134/1305. 


\title{
Charge redistribution and properties of high-temperature superconductors
}

\author{
Daniil I. Khomskii \\ Institute for Theoretical Physics, University of Cologne, D-5000 Köln 41, Federal Republic of Germany \\ and P. N. Lebedev Physical Institute, Moscow 117334, U.S.S.R. \\ Feodor V. Kusmartsev \\ Institute for Theoretical Physics, University of Cologne, D-5000 Köln 41, Federal Republic of Germany \\ and L. D. Landau Institute for Theoretical Physics, Moscow 117334, U.S.S.R.
}

(Received 29 May 1992)

\begin{abstract}
We show that in high- $T_{c}$ superconductors (HTSC) with two groups of electrons (e.g., holes in $\mathrm{CuO}_{2}$ planes and in a "reservoir") there should exist a charge redistribution with the temperature: the hole concentration $N_{h}$ in "active" superconducting $\mathrm{CuO}_{2}$ planes increases below $T_{c}$. This effect may explain structural changes such as the shift of the apical oxygen atom, anomalous thermal expansion, the shift of nuclear quadrupole resonance lines, the change of the positron lifetime, and the modification of the ion channeling below $T_{c}$. Some other possible consequences of the charge redistribution (the modification of the temperature dependence of a gap $\Delta$ and of the ratio $2 \Delta_{0} / T_{c}$, the phenomena at a contact of HTSC with normal metals and semiconductors) are discussed.
\end{abstract}

It is well known that the carrier concentration $N_{h}$ in "active" $\mathrm{CuO}_{2}$ planes is an important (albeit not the only) factor determining the critical temperature and other properties of the high- $T_{c}$ superconductors (HTSC) (see, e.g., Ref. 1). Most HTSC's consist of two electronic subsystems, namely, the $\mathrm{CuO}_{2}$ planes on the one hand and a "reservoir" on the other such as the chains in $\mathrm{YBa}_{2} \mathrm{Cu}_{3} \mathrm{O}_{7}$ or the $\mathrm{BiO}$ and $\mathrm{TlO}$ planes in $\mathrm{Bi}$ and Tl HTSC. ${ }^{2} \mathrm{~A}$ charge redistribution may occur between these two subsystems with changing external conditions. One of the most direct ways to determine the charge distribution and the valence state of the respective ions is provided by a detailed structural analysis. ${ }^{3}$ In particular, the position of the apex oxygen or the corresponding $\mathrm{Cu}-\mathrm{O}$ bond length is a sensitive probe of these effects.

Usually one discusses the charge redistribution with changing composition, such as oxygen stoichiometry, etc. Here we want to show that there should also occur a charge redistribution between these two subsystems with a change of temperature at fixed stoichiometry, especially that $N_{h}$ should increase when the system becomes superconducting. In principle, this effect has general character and is not specific for HTSC. However, because of the relatively large value of the ratio $\Delta / \epsilon_{F}$ of the HTSC's, the consequences should be more important and more easily detectable. Similar effects may also exist in some other systems, e.g., having metal-insulator transitions.

The charge redistribution as a possible source of certain experimentally observed anomalies in HTSC's at $T \leq T_{c}$ was mentioned previously in some experimental works (see Refs. 15 and 22 below). However, the physical nature of this charge redistribution was not discussed in any of them, the connection with the superconducting transition was not established, and it was not made clear that the effect has a general character.

A qualitative explanation of the effect is as follows. One may say that below $T_{c}$ there is an energy gain for the condensed holes in $\mathrm{CuO}_{2}$ planes. It should therefore be energetically favorable to transfer a part of the holes from the reservoir to the condensate region (here $\mathrm{CuO}_{2}$ planes) to make use of this condensation energy. To show this we should actually study the change of the chemical potential $\mu$ of the system, which determines the occupation of corresponding states.

In the traditional superconductors described by BCS theory the chemical potential is assumed to be fixed. This is a very good assumption for ordinary superconductors, where all the changes of $\mu \sim \epsilon_{F}$ with $T$ are small because $T_{c} / \epsilon_{F}<1$ (although there have been attempts to observe this shift even for these systems ${ }^{4}$ ). However, many unusual properties of the HTSC (the small coherence length $\xi$, the large ratio of $2 \Delta / T_{c}$, the often "wrong" temperature dependence of $\Delta$ etc.) cast some doubts on the applicability of a standard weak-coupling BCS theory to these materials. In some works (see, e.g., Ref. 5) the HTSC was even ascribed to a condensation of "preformed" bosons. Even if the limit of very tightly bound holes forming bosons does not apply, it may well be that the situation here is intermediate, so that the energy gap may not be much smaller than $\epsilon_{F}$. As we will show, in this case one can no longer neglect the temperature dependence of the chemical potential $\mu(T),{ }^{6,7}$ which may be large. This effect leads to quite interesting consequences, especially if there is also a hole reservoir. ${ }^{7}$

Let us first discuss a one-component system described by the usual BCS theory. In the normal state, the chemical potential is nearly constant at low temperatures,

$$
\mu(T)=\mu_{0}-T \exp \left(-\mu_{0} / T\right) .
$$

In a superconducting state the electron (or hole) distribution function $n(\xi)$ is given by the expression

$$
n(\xi)=\frac{1}{2}\left(1-\frac{\xi}{E} \tanh \frac{E}{2 T}\right),
$$


where $\xi(p)=\epsilon(p)-\mu$ and $E=\sqrt{\xi^{2}+\Delta^{2}} ; \epsilon(p)$ is the energy spectrum of free fermions.

From the equation for the total number of fermions $N=2 \int \rho(\xi) n(\xi) d \xi$ we can find the chemical potential $\mu(T)$. For a constant density of states $\rho(\epsilon)=\rho \theta(\epsilon) \theta(D-\epsilon)$ we get, at low temperatures and for $\Delta<\mu_{0}<D-\mu_{0}$,

$$
N=2 \rho\left(\mu+\frac{\Delta^{2}}{4 \mu}\right)
$$

from which we obtain

$$
\mu=\mu_{0}-\frac{\Delta^{2}}{4 \mu_{0}}
$$

If $\mu_{0}>\Delta / 2$, the term $-\Delta^{2} / 4 \mu_{0}$ in (4) is substituted by $+\Delta^{2} / 4\left(D-\mu_{0}\right)$.

The result (4) is obtained in the simplest model with a constant density of states $\rho(\epsilon)=$ const, with asymmetric position of the Fermi level. A more general expression may be easily obtained for arbitrary $\rho(\epsilon)$ :

$$
\mu=\mu_{0}-c \frac{\rho^{\prime}}{\rho} \Delta^{2}
$$

where the constant $c \sim 0.3, \rho^{\prime}$ is the derivative of the density of states at the Fermi level, and $\rho=\rho\left(\epsilon_{F}\right)$.

We see that, whereas in a normal state $\mu(T)$ increases with decreasing temperature [see (1)], in the SC state it starts to decrease. This is just the effect mentioned in the Introduction: the term $\sim \Delta^{2} / \mu_{0}=\Delta^{2} / \epsilon_{F}$ is the condensation energy.

If we have two electronic subsystems in the same materials, one becoming superconducting, and the other remaining normal, the transition of the first component to a SC state will be accompanied by the charge transfer from reservoir " 2 "' to the active subsystem " 1 ." If we ignore the exponential terms in $T$ and consider again the simplest case of the constant densities of states $\rho_{1}(\epsilon)$ and $\rho_{2}(\epsilon)$ for both subsystems, $\rho_{1}(\epsilon)=\rho_{1} \theta(\epsilon) \theta\left(D_{1}-\epsilon\right)$, $\rho_{2}(\epsilon)=\rho_{2} \theta(\epsilon-\delta) \theta\left(D_{2}+\delta-\epsilon\right)$, we get

$$
\begin{aligned}
& N_{1}=2 \rho_{1}\left(\mu+\frac{\Delta^{2}}{4 \mu}\right), \\
& N_{2}=2 \rho_{2}(\mu-\delta) \theta(\mu-\delta) .
\end{aligned}
$$

The condition of charge conservation $N_{1}+N_{2}=N$ $=$ const immediately gives us

$$
\mu=\mu_{0}-\frac{\rho_{1}}{\rho_{1}+\rho_{2}} \frac{\Delta^{2}}{4 \mu_{0}} \text {. }
$$

From (6) and (7) we find that the density of carriers in the SC subsystem increases below $T_{c}$ :

$$
N_{1}=N_{1}^{0}\left(1+\frac{\rho_{2}}{\rho_{1}+\rho_{2}} \frac{\Delta^{2}}{4 \mu_{0}^{2}}\right)
$$

where $N_{1}^{0}=2 \rho_{1} \mu_{0}$ is the carrier concentration in the first subsystem above $T_{c}$. The corresponding expression in the general case (5) has the form

$$
N_{1}=N_{1}^{0}+c \frac{\rho_{2}}{\rho_{1}+\rho_{2}} \rho_{1}^{\prime} \Delta^{2} .
$$

Thus, we see that in a two-component system, in which one subsystem becomes superconducting (as, e.g., $\mathrm{CuO}_{2}$ planes in HTSC), there should be a charge transfer from a reservoir into the SC subsystem. This effect is the strongest when the density of states of the reservoir $\rho_{2}$ is large (therefore the effect will still be presented and will even become stronger if the electron states in a reservoir are localized). The effect is also enhanced if the density of states changes sharply close to $\epsilon_{F}$.

Crudely, the net relative change of carrier concentration will be of order $\left(\Delta / \epsilon_{F}\right)^{2}$. Therefore, it can be ignored in an ordinary SC [although there were reports of a change of chemical potential and work function even for Sn (Ref. 4)]. In the HTSC, two factors can make the situation quite different: First, here there are really two electronic, or hole, subsystems within the same material, which makes the process of charge redistribution a bulk effect (this is also the case in superlattices of SC and normal metals). Second, and more importantly, in these systems $\Delta$ is much larger than in the ordinary SC. One can estimate the value of the effect from Eq. (9), taking the measured value of $\Delta \simeq 300 \mathrm{~K}$ and using the values of $\rho^{\prime}$ and $\rho$ obtained in the band-structure calculation, ${ }^{24}$ $\rho^{\prime} / \rho \sim 10^{-3} \mathrm{~K}^{-1}$. One gets the change of the hole concentration (per elementary cell) of order $10^{-2}$. The increase of the hole concentration in the $\mathrm{CuO}_{2}$ planes below $T_{c}$ may thus reach several percents, which should already have detectable consequences.

One should also take into account the Coulomb interaction $W \sim e^{2} / \varepsilon d$ between the holes in a reservoir and in the $\mathrm{CuO}_{2}$ plane ( $d$ is the mean distance between them). Carrying out the calculations similar to the previous ones we finally get

$$
N_{1}=N_{1}^{0}\left[1+\frac{\rho_{2}}{\rho_{1}+\rho_{2}} \frac{\Delta^{2}}{4 \mu_{0}^{2}\left\{1+\left[4 \rho_{1} \rho_{2} /\left(\rho_{1}+\rho_{2}\right)\right] W\right\}}\right] .
$$

If the average Coulomb energy $W \ll \epsilon_{F} \sim \mu_{0}$, we recover the result (8) (recall that $\rho \sim \epsilon_{F}^{-1}$ ). If on the other hand $W \gg \epsilon_{F}$, then

$$
N_{1}=N_{1}^{0}\left(1+\frac{\Delta^{2}}{16 \mu_{0} W}\right) \text {. }
$$

The estimates show that $W \sim 0.2-0.4 \mathrm{eV}$, so the Coulomb interaction does not change qualitatively the degree of charge redistribution below $T_{c}$.

In the above discussion we have used the standard BCS theory. The applicability of this approach to the HTSC is somewhat questionable. We can show, however, that the main effect remains qualitatively the same, irrespective of the detailed description of the SC state. One can see it even in the opposite limit of tightly bound local pairs. ${ }^{7}$

Let us now discuss some experimental consequences of the effect of charge redistribution. As mentioned above, the most direct way to get information on the charge dis- 
tribution in crystals is a careful structural study, including the measurement of separate bond lengths and of the bond valence sums $\sigma$. The results of Ref. 3 show that $T_{c}$ in 1:2:3 HTSC scales with $N_{h}$ determined by $\sigma[\mathrm{Cu}(2)]$. The main contribution to the change of $\sigma$ comes from a shift of the apex oxygen from the chains towards the planes with increasing oxygen content.

The strong shift of the apex oxygen toward $\mathrm{CuO}_{2}$ planes below $T_{c}$ in $\mathrm{Bi}-2: 2: 1: 2$ and $\mathrm{Bi}$ 2:2:2:3 HTSC compounds was observed in Ref. 8 . There are, in principle, two possibilities to explain such a shift. One of them is just the net charge redistribution discussed above: the total increase of the hole concentration $N_{h}$ below $T_{c}$ in an "active" $\mathrm{CuO}_{2}$ plane should lead to just such a shift of the apex $\mathrm{O}(4)$. Another possibility to explain this shift of $\mathrm{O}(4)$ is a redistribution of an already existing charge, i.e., a promotion of the $\mathrm{Cu}^{2+} d$ hole from the $d_{x^{2}-y^{2}}$ to the $d_{z^{2}}$ orbital. ${ }^{9}$ Actually these two factors may act together, while the primary source seems to be an increase of $N_{h}$ below $T_{c} \cdot{ }^{10}$

On the other hand, for the 1:2:3 HTSC compounds the situation is rather controversial. Some of the earlier neutron data ${ }^{11}$ have shown similar behavior of the $\mathrm{Cu}(2)$ $\mathrm{O}(4)$ and $\mathrm{Cu}(1)-\mathrm{O}(4)$ distances below $T_{c}$, as in the above cases. However, in recent neutron diffraction work ${ }^{12}$ no effect at $T_{c}$ was noticed, although the gross behavior observed over a wide temperature range, e.g., an increase of the $\mathrm{Cu}(1)-\mathrm{O}(4)$ bond length with decreasing temperature, may be naturally explained by the effects discussed above.

At the same time extended x-ray-absorption finestructure (EXAFS) data ${ }^{13,14}$ show that there is an effect in the temperature range $80-110 \mathrm{~K}$. One should note that EXAFS gives more local information than neutron diffraction; even strong distortions having only shortrange order may remain undetected in diffraction studies like the one by Kwei et al. ${ }^{12}$ However, they may be probed by the neutron studies of the pair distribution function-see Ref. 15, where the marked structural changes, possibly of local nature, were observed in $\mathrm{Tl}$ HTSC below $T_{c}$. As one of the possible explanations the authors of Ref. 15 mention the charge redistribution (however, without an explanation of why it should occur at $T_{c}$ ). One should also notice that the detailed pattern of the distortions observed in Ref. 15 is difficult to explain using only the kind of the charge redistribution described in our paper; probably some extra factors are important here.

The effect of charge redistribution with temperature may also lead to strong anomalies of the thermal expansion $\alpha$. Thus, the anomalous and anisotropic behavior of $\alpha$ close to $T_{c}$, observed in untwinned $1: 2: 3$ crystals, ${ }^{16}$ may be naturally explained by the decrease of the hole concentration in chains: it would lead to the decrease of the lattice parameter $b$ below $T_{c}$, consistent with experimental observations.

Another sensitive local probe of a charge redistribution and of the connected structural distortions is NQR. Such studies have shown that there is a shift of the NQR frequency of $\mathrm{Cu}(1)$ (Ref. 17 and 18) and of Ba (Ref. 19) in
$\mathrm{YBa}_{2} \mathrm{Cu}_{3} \mathrm{O}_{7}$, the character of which changes markedly at $T_{c}$.

One more experimental result also seems to be consistent with our picture. The increase of the hole concentration in the $\mathrm{CuO}_{2}$ plane below $T_{c}$ may lead to an extra screening of the interatomic forces and can thus explain strong phonon softening at $T_{c}$ for the vibrations in $\mathrm{Cu}-\mathrm{O}$ planes in $\mathrm{Bi} 2: 2: 1: 2{ }^{20}$ At the same time the transfer of holes from chains to planes in 1:2:3 and the corresponding shift of the apical oxygen atom $\mathrm{O}(4)$ changes the coupling in the $\mathrm{Cu}(2)-\mathrm{O}(4)-\mathrm{O}(1)$ row: if above $T_{c}$ the strongly coupled $\mathrm{Cu}(1)-\mathrm{O}(4)$ unit vibrates relatively independently from the distant $\mathrm{Cu}(2)$, the "more homogeneous" situation below $T_{c}$ may lead to the more coherent motion of these atoms which can explain strong anomalies in ion channeling at $T_{c} \cdot{ }^{21}$

Probably the most direct confirmation of the effect of charge redistribution below $T_{c}$ comes from the recent study of positron annihilation in $\mathrm{YBa}_{2} \mathrm{Cu}_{3} \mathrm{O}_{7}{ }^{22}$ The decrease of positron lifetime below $T_{c}$ was attributed in this work to a "local charge transfer between $\mathrm{CuO}_{2}$ layers and $\mathrm{Cu}-\mathrm{O}$ chains as the materials become superconducting." The effect observed in Ref. 22 for undoped $\mathrm{YBa}_{2} \mathrm{Cu}_{3} \mathrm{O}_{7}$ is of order $7 \%$, consistent with our estimates.

Yet another result, consistent with our picture, was obtained recently in Ref. 25: It was observed in the study of $O K$-edge absorption spectra that the concentration of holes in $\mathrm{CuO}_{2}$ planes increases below $T_{c}$, and the effect goes away when the superconductivity is suppressed by high current.

The effect discussed above may also lead to other observable consequences. If $N_{h}(T)$ changes significantly at $T<T_{c}$, the temperature dependence of all thermodynamic properties of the HTSC in the SC state will be modified. For instance, in the case of not very heavily doped systems, $T_{c}$ and $\Delta$ are proportional to $N_{h}$. ${ }^{1}$ Therefore, one should expect a change of the temperature dependence of $\Delta(T)$ and of the ratio $2 \Delta(0) / T_{c}$. Indeed, if we take into account the dependence (8 and 9): $N_{h}(T=0)=N_{h}^{0}\left[1+\gamma \Delta^{2}(0)\right]$, and take also $T_{c}\left(N_{h}\right)=\alpha N_{h}$ then we get, at low temperatures

$$
\Delta^{2}(0)=\frac{\Delta_{0}^{2}}{1-\gamma \Delta_{0}^{2}} .
$$

Here $\Delta_{0}$ is the gap at $T=0$ in the absence of charge redistribution at a constant concentration of holes in the $\mathrm{CuO}_{2}$ planes $N_{h}^{0}$. One sees that due to the increase of $N_{h}$ with decreasing temperature $\Delta(T)$ does increase faster than would be expected for a given $T_{c}$ in the BCS limit, and $2 \Delta(0) / T_{c}$ becomes larger than the BCS value 3.5. The magnitude of this effect depends on the parameters of the HTSC. In order to get the observed values of $2 \Delta(0) / T_{c} \sim 6-7$, the value of $N_{h}(T=0)$ should be approximately twice as large as at $T_{c}$. In the actual HTSC this is hardly the case; the increase of the hole density is probably at most, $\sim 5-10 \%$. So some other mechanisms of the increase of this ratio must be at work here, e.g., strong-coupling effects or the suppression of the superconductivity by thermally excited phonons (see, e.g., Ref. 
23). However, qualitatively the charge redistribution modifies the dependence $\Delta(T)$ "in the right direction."

Several interesting phenomena may occur due to the effects discussed above on the contact of a HTSC with ordinary metals or superconductors. The change of the chemical potential of HTSC's below $T_{c}$ may lead to a charge transfer at the contact and to a modification of the contact barrier. This may have some consequences for the tunneling process, for thermoelectric effects, etc. The effect is, in principle, universal and must also exist for ordinary superconductors, but it will be more significant in the HTSC due to the very large condensation energy.

The contact of a HTSC with a semiconductor might be more promising for the observation of the discussed effects. The change of $\mu(T)$ and the corresponding charge transfer may induce a voltage drop in a surface layer of a semiconductor and significantly change its properties. Such changes can be very precisely detected.

One more comment concerns the possible existence of similar phenomena in other systems in which the gap is formed in the electronic spectrum, e.g., in the systems with metal-insulator transitions. Quite often such transitions are very sharp, with a jump in conductivity up to
$10^{7}$. Consequently, one may expect strong effects of the type discussed above, e.g., on a contact of such materials with standard metals or semiconductors or in corresponding superlattices.

To summarize, in this work we have shown that there should be a charge redistribution accompanying the formation of a gap in the electronic spectrum, notably in HTSC's, either between electronic subsystems within the same material (e.g., $\mathrm{CuO}$ chains and $\mathrm{CuO}_{2}$ planes) or on contacts of such materials with ordinary metals and semiconductors. An increase of the hole density in the $\mathrm{CuO}_{2}$ planes in a SC phase may naturally explain many anomalies observed in the HTSC at and below $T_{c}$. Some other consequences of this phenomenon are also discussed and it is concluded that similar effects may, in principle, also be observable in other materials, e.g., having metal-insulator transitions.

The authors express their gratitude for useful discussion to Professor V. Emery, Professor H. Lütgemeier, and Professor D. Wohlleben, and to Dr. B. Büchner, Dr. A. Freimuth, and especially to Dr. J. Röhler.
${ }^{1}$ Y. F. Uemura et al., Phys. Rev. Lett. 62, 2317 (1989).

${ }^{2}$ The exception might be $\mathrm{La}_{2-x} M_{x} \mathrm{CuO}_{4}$, although recent calculations by R. P. Gupta and M. Gupta [Physica C 185-189, 1599 (1991)] show that even in this system there may exist charge reservoir-in this case its role is played by rare-earth ions.

${ }^{3}$ R. J. Cava et al., Physica C 165, 419 (1990).

${ }^{4}$ M. Shott and A. J. Walton, Phys. Lett. A 60, 53 (1977).

${ }^{5}$ R. Micnas, J. Ranninger, and S. Robaszkiewicz, Rev. Mod. Phys. 62, 113 (1990).

${ }^{6}$ D. van der Marel, Physica C 165, 35 (1990).

${ }^{7}$ D. I. Khomskii and F. V. Kusmartsev, Pis'ma Zh. Eksp. Teor. Fiz. 54, 150 (1991); in Physics and Material Science of High Temperature Superconductors II, Vol. 209 of NATO Advanced Study Institute, Series E, edited by R. Kossowsky, B. Raveau, D. Wohlleben, and S. Patapis (Kluwer Academic, Dordrecht, 1992), p. 65.

${ }^{8} \mathrm{~J}$. Röhler and A. Larisch, in Electronic Properties of High $-T_{c}$ Superconductors and Related Compounds, edited by $\mathrm{H}$. Kusmany, M. Mehring, and J. Fink, Springer Series in Solid State Sciences Vol. 99 (Springer-Verlag, Berlin, 1990), p. 152.

${ }^{9}$ D. I. Khomskii, Physica B 171, 44 (1991).

${ }^{10}$ D. I. Khomskii and E. I. Neimark, Physica C 173, 342 (1991).
${ }^{11}$ M. François et al., Solid State Commun. 66, 1117 (1988).

${ }^{12}$ G. H. Kwei et al., Physica C 169, 217 (1990).

${ }^{13}$ S. D. Conradson and I. D. Raistrick, Science 243, 1340 (1989).

${ }^{14}$ J. Röhler et al., Physica C 191, 57 (1991).

${ }^{15}$ B. H. Toby et al., Phys. Rev. Lett. 64, 2414 (1990).

${ }^{16}$ C. Meingast et al., Phys. Rev. Lett. 67, 1634 (1991).

${ }^{17} \mathrm{H}$. Riesemeier et al., in Electronic Properties of $\mathrm{High}-\mathrm{T}_{c} \mathrm{Su}$ perconductors and Related Compounds, edited by $\mathrm{H}$. Kusmany, M. Mehring, and J. Fink, Springer Series in Solid State Sciences Vol. 99 (Springer-Verlag, Berlin, 1990), p. 225.

${ }^{18}$ D. Brinkmann, in Electronic Properties of High- $T_{c}$ Superconductors and Related Compounds, edited by H. Kusmany, M. Mehring, and J. Fink, Springer Series in Solid State Sciences Vol. 99 (Springer-Verlag, Berlin, 1990), p. 195.

${ }^{19} \mathrm{H}$. Lütgemeier et al. (unpublished).

${ }^{20}$ H. J. Mook et al., Phys. Rev. Lett. 65, 2712 (1990).

${ }^{21}$ R. P. Sharma et al., Physica C 174, 409 (1991).

22J. C. Jean et al., Phys. Rev. Lett. 64, 1593 (1990). We are grateful to Dr. R. Monnier for pointing out this reference to us.

${ }^{23}$ D. I. Khomskii, Phys. Lett. A 138, 435 (1989).

${ }^{24}$ I. I. Mazin (private communication).

${ }^{25}$ Y. Hirai et al., Phys. Rev. B 45, 2573 (1992). 